Reprod. Nutr. Dévelop., 1986, 26 (3), 815-826.

\title{
Effets de la prolactine sur la sécrétion des lipides du lait dans les cellules épithéliales mammaires de lapine en lactation
}

\author{
Michèle OLLIVIER-BOUSQUET, Y. DEMARNE (*)
}

avec la collaboration technique de Georgette GIANNI et Janette PIHET

Laboratoire de Physiologie de la Lactation,

(*) Station de Recherches de Nutrition,

I.N.R.A., 78350 Jouy-en-Josas, France.

Summary. Effect of prolactin on milk lipid secretion in lactating rabbit mammary gland epithelial cells.

Milk lipids of lactating rabbit mammary epithelial cells were labelled in vitro during a 3min pulse with ${ }^{14} \mathrm{C}-\mathrm{Na}$ butyrate. After $1 \mathrm{~h}$ of incubation in the presence or not of prolactin, the different classes of lipids, in the tissues and the incubating mediums, were separated by thin-layer chromatography. The radioactivity of spots corresponding to the different classes of lipids (compared with the standards) was counted by liquid scintillation.

In the tissues, triglycerides were the most strongly labelled constituent $197.7 \%$ of the radioactivity was bound to triglycerides). In the medium, triglyceride radioactivity was less ; only $70.9 \%$ was bound to the triglycerides.

In the presence of prolactin $(10 \mu \mathrm{g} / \mathrm{ml})$ the distribution of radioactivity in the tissues was not modified but in the medium, secreted triglycerides were more radioactive than in the control medium.

In the presence of protamine sulfate $(100 \mu \mathrm{g} / \mathrm{ml})$, an inhibitor of lipoprotein-lipase, radioactivity in the secreted triglycerides decreased less, suggesting that this decrease was due to hydrolysis by lipoprotein lipase, probably during the process of exocytosis.

Morphological evaluation on electron micrographs of the number of intracellular and extracellular lipid globules showed that prolactin increased the number of extracellular lipid globules; this suggested a higher secretion.

These results show that the radioactivity of neosynthesized lipid constituents was modified during the secretion of milk lipids. Prolactin, which increased the total labelled lipids secreted, had an effect on the distribution of the radioactivity of the different lipid classes.

Les lipides du lait sont sécrétés par la cellule mammaire sous forme de globules entourés d'une membrane provenant de la membrane apicale de la cellule (" Milk fat globule membrane »: MFGM). De nombreux travaux ont permis de préciser la nature, la composition et les caractéristiques des graisses du lait et de la MFGM, et de décrire les étapes de libération des globules lipidiques (voir revue 
Keenan et Dylewski, 1985). La prolactine joue un rôle important dans le maintien de la sécrétion du lait (Cowie et Tindal, 1971). Un effet rapide et direct de la prolactine sur la sécrétion lipidique a été mis en évidence (Daudet et al., 1981). Cette hormone provoque en effet une augmentation de la libération des lipides néosynthétisés marqués au cours d'un "pulse ".

Afin d'étudier plus précisément le mécanisme de cette stimulation prolactinique, nous avons analysé la distribution de la radioactivité parmi les différents constituants des lipides néosynthétisés. De plus, nous avons évalué morphologiquement en microscopie électronique l'effet de I'hormone sur la répartition intraet extracellulaire des globules lipidiques.

\section{Matériel et méthodes.}

Animaux. - Des lapines néo-zélandaises dont les portées ont été ramenées à 8 petits, sont abattues entre le $14^{\circ}$ et le $20^{\circ}$ jour de lactation, $3 \mathrm{~h}$ après la dernière tétée.

Incubations. - Des fragments de tissu mammaire $10,5 \mathrm{~mm}$ de côté environ): poids total de l'échantillon par essai : 30 à $50 \mathrm{mg}$ ) sont incubés dans un milieu de Krebs Ringer bicarbonaté $\left(\mathrm{pH} 7,4 ; 37^{\circ} \mathrm{C}\right.$; atmosphère $95 \% \mathrm{O}_{2}, 5 \% \mathrm{CO}_{2}$; glucose $1 \mathrm{~g} / 1)$.

Après une préincubation de 10 à $30 \mathrm{~min}$, les fragments sont marqués au cours d'un " pulse » $\left(3 \mathrm{~min}\right.$ ) par $50 \mu \mathrm{Ci} / \mathrm{ml}$ de butyrate de $\mathrm{Na}^{14} \mathrm{C}-3-4$ (CEA Saclay, $25 \mathrm{MCi} / \mathrm{mM}$ ). Après le marquage, les tissus sont rincés abondamment puis remis à incuber dans un milieu de Krebs Ringer bicarbonaté en présence ou non de prolactine $(10 \mu \mathrm{g} / \mathrm{ml})$ et de sulfate de protamine $(100 \mu \mathrm{g} / \mathrm{ml})$.

Dans le but d'étudier l'activité des lipoprotéines lipases qui pourraient être sécrétées dans le milieu, certains fragments de tissu mammaire (30 à 50 mg par essai) sont incubés dans un milieu de Krebs Ringer bicarbonaté et glucosé comme précédemment. Après une heure d'incubation, les milieux sont récupérés et débarrassés des débris cellulaires par une centrifugation (1 $500 \mathrm{t}, 5 \mathrm{~min}$ ). $5 \mu \mathrm{Ci} / \mathrm{ml}$ de trioléine ${ }^{14} \mathrm{C}$ (CEA Saclay, $50-80 \mathrm{mCi} / \mathrm{mM}$ ) émulsionnée dans du lipiphysan $10 \%$ (Egic) sont rajoutés au milieu et l'incubation $\left(37{ }^{\circ} \mathrm{C}, 95 \% \mathrm{O}_{2}, 5 \% \mathrm{CO}_{2}\right)$ est prolongée pendant une heure.

Extraction des lipides. - Les lipides sont extraits à partir des milieux selon la technique de Folch et al. (1957). L'extrait est séché par addition de sulfate de sodium, filtré et évaporé. La radioactivité des lipides tissulaires est déterminée selon le même protocole, après broyage dans le mélange chloroforme-méthanoleau $(3: 4: 8) \mathrm{v} / \mathrm{v}$.

Chromatographie sur couche mince. - Pour séparer les différents constituants lipidiques à partir des lipides totaux, des chromatographies sur couche mince sont réalisées sur des plaques de gel de silice (Merck 60) et développées dans un mélange hexane/éther/acide formique $(85: 20: 2) \mathrm{v} / \mathrm{v}$, puis hexane/éther/méthanol/acide formique $(40: 60: 0,8: 0,08) \mathrm{v} / \mathrm{v}$ et révélées à l'iode. Les différentes taches, identifiées à l'aide de standards, sont grattées et comptées en présence 
de gel (Thixotropic Gel Powder, CAB-O-SIL, Packard, $40 \mathrm{mg} / \mathrm{ml}$ ) et de mélange scintillant dans un compteur à scintillation liquide (Packard 460-c). La radioactivité de chaque constituant est calculée en $\%$ de la radioactivité totale.

Microscopie électronique. - Les tissus sont fixés dans un mélange paraformaldéhyde $4 \%$, glutaraldéhyde $4 \%(\mathrm{v} / \mathrm{v})$ dans un tampon phosphate $0,1 \mathrm{M}(\mathrm{pH}$ 7,25), puis dans du tétroxyde d'osmium dilué à $1 \%$ dans le même tampon. Ils sont ensuite déshydratés dans l'éthanol, contrastés en bloc par l'acétate d'uranyle $4 \%$, puis inclus dans l'épon. Les coupes sont contrastées par le citrate de plomb $1 \%$. Sur 240 micrographies ( $\times 5000$ à 8000 ) comprenant une ou plusieurs cellules épithéliales et une partie adjacente de la lumière de l'acinus, le nombre de globules lipidiques intracellulaires et extracellulaires a été compté.

Analyse statistique des résultats. - L'analyse statistique des résultats a été faite par le test $t$ de Student sur les différences appariées des couples de valeur provenant du même animal. Les comparaisons ont été faites d'une part entre les valeurs des différents constituants des lipides intracellulaires et sécrétés dans les milieux, d'autre part entre les valeurs des différents constituants des lipides sécrétés dans les milieux d'incubation avec ou sans prolactine.

\section{Résultats.}

Répartition de la radioactivité dans les différentes classes de lipides. - Conformément aux expériences décrites précédemment (Daudet et al., 1981), le butyrate de sodium est incorporé et estérifié dans les lipides des fragments de glande mammaire in vitro. En effet, après une heure d'incubation, les lipides totaux des tissus sont marqués $(13833 \pm 1300 \mathrm{cpm} / \mathrm{mg}$ de tissu, $n=8$ ).

La répartition de la radioactivité dans les différentes classes de lipides des tissus et des milieux est montrée dans le tableau 1 (tissus témoins et milieux témoins). II apparaît clairement que les triglycérides constituent la classe de lipides la plus fortement marquée aussi bien dans les tissus que dans les milieux. II apparaît de plus que la répartition de la radioactivité dans les lipides du milieu est différente de celle des tissus. En effet, la radioactivité des triglycérides est significativement moins élevée que dans les tissus alors que la radioactivité des phospholipides, des diglycérides et des acides gras est significativement plus élevée. Les différences entre les valeurs de radioactivité des monoglycérides et des esters du cholestérol, bien que plus élevées dans les milieux que dans les tissus, ne sont pas statistiquement significatives.

Effet de la prolactine sur la répartition de la radioactivité dans les différentes classes de lipides. - La radioactivité des fragments marqués par le butyrate de sodium puis incubés pendant une heure en présence de prolactine n'est pas modifiée d'une façon statistiquement significative par rapport à celle des fragments incubés dans un milieu témoin (12 745 $\pm 1124 \mathrm{cpm} / \mathrm{mg}$ de tissu, $\mathrm{n}=8$ ), ce qui est en accord avec les expériences décrites précédemment (Daudet et al., 1981). De plus, la répartition de la radioactivité dans les différentes classes de lipides du tissu n'est pas modifiée par rapport à celle des tissus témoins (tabl. 1 : Tissus Témoin ; Tissus-Prolactine). La répartition de la radioactivité des différentes clas- 
TABLEAU 1

Répartition de la radioactivité $(\%)$ dans les différentes classes de lipides des fragments de tissus mammaires et dans les lipides sécrétés dans le milieu d'incubation.

\begin{tabular}{|c|c|c|c|c|}
\hline & \multicolumn{2}{|c|}{$\begin{array}{l}\text { Répartition de la radioactivité } \\
\text { dans les tissus }\end{array}$} & \multicolumn{2}{|c|}{$\begin{array}{l}\text { Répartition de la radioactivité } \\
\text { dans les milieux }\end{array}$} \\
\hline & Témoin & $\begin{array}{l}\text { Prolactine } \\
(10 \mu \mathrm{g} / \mathrm{ml})\end{array}$ & Témoin & $\begin{array}{l}\text { Prolactine } \\
(10 \mu \mathrm{g} / \mathrm{ml})\end{array}$ \\
\hline Phospholipides & $0,23 \pm 0,04$ & $0,30 \pm 0,07$ & $13,14 \pm 4,78^{\mathrm{a}^{* *}}$ & $8,95 \pm 4,34^{e^{* \cdots *}}$ \\
\hline Monoglycérides & $0,18 \pm 0,02$ & $0,18 \pm 0,04$ & $10,45 \pm 5,02$ & $7,55 \pm 4,36$ \\
\hline Diglycérides & $0,65 \pm 0,09$ & $0,64 \pm 0,04$ & $3,92 \pm 0,71^{\mathrm{b}^{* *}}$ & $2,98 \pm 0,34^{4 *}$ \\
\hline Acides gras & $0,48 \pm 0,1$ & $0,40 \pm 0,05$ & $4,35 \pm 1,45^{c^{*}}$ & $2,64 \pm 0,77^{9^{*}}$ \\
\hline Triglycérides & $97,70 \pm 0,39$ & $97,38 \pm 0,53$ & $70,94 \pm 6,35^{\mathrm{d}^{* \cdots}}$ & $81,40 \pm 6,04 \%: *$ \\
\hline $\begin{array}{l}\text { Esters } \\
\text { du cholestérol }\end{array}$ & $0,75 \pm 0,39$ & $0,12 \pm 0,04$ & $8,35 \pm 3,77$ & $1,76 \pm 1,04$ \\
\hline
\end{tabular}

Les fragments sont marqués pendant $3 \mathrm{~min}$ par $50 \mu \mathrm{Ci} / \mathrm{ml}$ de butyrate de sodium ${ }^{14} \mathrm{C}$, rincés abondamment puis incubés pendant une heure soit dans un milieu témoin, soit dans un milieu contenant $10 \mu \mathrm{g} / \mathrm{ml}$ de prolactine. Les tissus sont broyés dans un mélange chloroforme-méthanol-eau (3 : $4: 8$ ). Les lipides du milieu sont extraits dans le même mélange.

Les lipides extraits sont séparés par chromatographie sur couche mince et la radioactivité des taches comptée par scintillation liquide. Les résultats sont exprimés en \% de la radioactivité totale des lipides. $(\mathrm{n}=9$ ).

${ }^{*} \mathrm{p}<0,05 ;{ }^{* *} \mathrm{p}<0,02 ;{ }^{* * *} \mathrm{p}<0,01 ;{ }^{* * * *} \mathrm{p}<0,001$.

$a, b, c, d=$ comparaison avec les valeurs correspondantes des classes de lipides contenus dans les tissus témoins; $e, f, g, h=$ comparaison avec les valeurs correspondantes des classes de lipides contenus dans les tissus incubés en présence de prolactine $; i=$ comparaison entre les triglycérides sécrétés après incubation dans un milieu témoin et en présence de prolactine.

ses de lipides sécrétés en présence de prolactine est différente de celle des lipides contenus dans les tissus (tabl. 1 : Tissus-Prolactine; Milieux-Prolactine). La radioactivité des triglycérides est significativement plus faible dans le milieu et la radioactivité des phospholipides, des diglycérides et des acides gras est plus élevée.

On observe donc aussi une différence de répartition de la radioactivité dans les différentes classes de lipides entre les lipides contenus dans les tissus et les lipides sécrétés, en présence de l'hormone.

Cependant, la comparaison entre la répartition de la radioactivité des lipides sécrétés dans le milieu témoin et des lipides sécrétés en présence de prolactine montre que la radioactivité des triglycérides est significativement plus élevée en présence de prolactine. Les radioactivités des phospholipides, des acides gras et des esters du cholestérol, bien que plus faibles, ne sont pas significativement différentes de celles mesurées dans les milieux témoins.

En conclusion, ces résultats montrent que les lipides sécrétés ont une composition en triglycérides radioactifs modifiée par rapport à celle des lipides intra- 
cellulaires. En présence de prolactine, la radioactivité associée aux triglycérides sécrétés est augmentée.

Effet de la lipoprotéine lipase sur les lipides sécrétés. - La lipoprotéine lipase (LPL) catalyse l'hydrolyse qui libère les acides gras des triglycérides. Cette enzyme a été mise en évidence dans le lait et dans les cellules épithéliales mammaires où elle joue un rôle important dans la lipogenèse (Clegg, 1982). La diminution relative de la radioactivité observée dans les triglycérides sécrétés dans le milieu pourrait être le résultat d'une hydrolyse partielle due à cette enzyme. Afin de vérifier cette hypothèse, deux types d'expériences ont été réalisées.

1) Les fragments de tissu mammaire ont été incubés dans un milieu de Krebs Ringer bicarbonaté et glucosé, en présence d'un inhibiteur de la LPL, le sulfate de protamine $(100 \mu \mathrm{g} / \mathrm{ml})$ (Clegg, 1981). Cet inhibiteur ne provoque pas de modification de la radioactivité des différentes classes de lipides intracellulaires (tabl. 2). Au contraire, dans le milieu, en présence de sulfate de protamine, la radioactivité relative des triglycérides est augmentée. Le sulfate de protamine ne modifie pas l'effet de la prolactine (tabl. 3).

2) Un deuxième type d'expérience a été réalisé afin de savoir si une lipolyse est due à la LPL déjà sécrétée dans le milieu d'incubation. Pour cela, des milieux ont été recueillis après $1 \mathrm{~h}$ d'incubation en présence de fragments de tissus mammaires. Après avoir éliminé les tissus, $5 \mu \mathrm{Ci} / \mathrm{ml}$ de trioléine ${ }^{14} \mathrm{C}$ ont été rajoutés. Après une heure, la radioactivité des différentes classes de lipides a été mesurée dans le milieu. Le tableau 4 montre que l'hydrolyse des triglycérides dans ces conditions est très limitée et ne peut pas expliquer la forte diminution de radioactivité des triglycérides sécrétés.

TABLEAU 2

Répartition de la radioactivité dans les tissus.

\begin{tabular}{lcccc}
\hline & Témoin & $\begin{array}{c}\text { Témoin }+ \text { Sulfate } \\
\text { de protamine } \\
\left(100 \mu^{\prime} \mathrm{g} / \mathrm{ml}\right)\end{array}$ & $\begin{array}{c}\text { Prolactine } \\
(10 \mu \mathrm{g} / \mathrm{ml})\end{array}$ & $\begin{array}{c}\text { Prolactine }(10 \mu \mathrm{g} / \mathrm{ml}) \\
+\begin{array}{c}\text { Sulfate } \\
\text { de protamine } \\
(100 \mu \mathrm{g} / \mathrm{ml})\end{array}\end{array}$ \\
\hline Phospholipides & 0,45 & 0,26 & 0,35 & 0,24 \\
\hline Monoglycérides & 0,21 & 0,14 & 0,22 & 0,09 \\
\hline Diglycérides & 0,84 & 1,14 & 1,27 & 0,35 \\
\hline Acides gras & 0,54 & 0,52 & 0,60 & 0,49 \\
\hline Triglycérides & 97,46 & 97,94 & 97,56 & 98,83 \\
\hline
\end{tabular}

Répartition de la radioactivité $(\%)$ dans les différentes classes de lipides de fragments de tissus mammaires incubés dans un milieu de Krebs Ringer bicarbonaté glucosé. Les fragments sont marqués pendant 3 min par $50 \mu \mathrm{Ci} / \mathrm{ml}$ de butyrate de sodium ${ }^{14} \mathrm{C}$, rincés abondamment puis incubés pendant une heure soit dans un milieu de Krebs Ringer, soit dans un milieu de Krebs Ringer $+10 \mu \mathrm{g} / \mathrm{ml} \mathrm{de}$ prolactine, en présence ou non de sulfate de protamine $(100 \mu \mathrm{g} / \mathrm{ml})$. Les tissus sont broyés dans un mélange chloroforme-méthanol-eau $(3: 4: 8)$. Les lipides extraits sont séparés par chromatographie sur couche mince et la radioactivité des taches est comptée en scintillation liquide. Les résultats sont exprimés en \% de la radioactivité totale (résultat d'une expérience. Deux autres expériences faites avec des précurseurs différents donnent des résultats identiques). 
L'ensemble de ces résultats montre que la LPL provoque une hydrolyse partielle des triglycérides des lipides sécrétés. Cependant, cette hydrolyse ne paraît pas avoir lieu pendant le séjour des lipides dans le milieu d'incubation. De plus, elle n'a pas lieu lorsque les fragments sont incubés en présence de prolactine.

\section{TABLEAU 3}

Répartition de la radioactivité dans les milieux.

\begin{tabular}{lcccc}
\hline & Témoin & $\begin{array}{c}\text { Témoin + Sulfate } \\
\text { de protamine } \\
(100 \mu \mathrm{g} / \mathrm{ml})\end{array}$ & $\begin{array}{c}\text { Prolactine } \\
(10 \mu \mathrm{g} / \mathrm{ml})\end{array}$ & $\begin{array}{c}\text { Prolactine }(10 \mu \mathrm{g} / \mathrm{ml}) \\
+\begin{array}{c}\text { Sulfate } \\
\text { de protamine } \\
(100 \mu \mathrm{g} / \mathrm{ml})\end{array}\end{array}$ \\
\hline Phospholipides & 18,73 & 7,45 & 8,45 & 10,86 \\
\hline Monoglycérides & - & - & - & - \\
\hline Diglycérides & - & 1,94 & - & 6,32 \\
\hline Acides gras & 12,68 & 3,68 & 9,71 & 82,82 \\
\hline Triglycérides & 68,69 & 86,90 & 81,81 &
\end{tabular}

Répartition de la radioactivité dans les différentes classes de lipides sécrétés par des fragments de tissus mammaires incubés dans un milieu de Krebs Ringer bicarbonaté glucosé. Les tragments sont marqués pendant $3 \mathrm{~min}$ par $50 \mu \mathrm{Ci} / \mathrm{ml}$ de butyrate de sodium ${ }^{14} \mathrm{C}$, rincés abondamment puis incubés pendant une heure soit dans un milieu témoin, soit dans un milieu contenant $10 \mu \mathrm{g} / \mathrm{ml}$ de prolactine, en présence ou non de $100 \mu \mathrm{g} / \mathrm{ml}$ de sulfate de protamine. Les lipides du milieu sont extraits par un mélange chloroforme-méthanol-eau $(3: 4: 8)$ et séparés par chromatographie sur couche mince. La radioactivité des taches est comptée en scintillation liquide (résultat d'une expérience. Deux autres expériences faites avec des précurseurs différents donnent des résultats identiques).

TABLEAU 4

Analyse de la radioactivité dans un milieu d'incubation ayant au préalable contenu des fragments de tissu mammaire, apres une heure en présence de trioléate

${ }^{14} \mathrm{C}(5 \mu \mathrm{Ci} / \mathrm{ml})$.

\begin{tabular}{lc}
\hline & $\begin{array}{c}\text { Radioactivité } \\
\text { (\% de la radioactivité totale) }\end{array}$ \\
\hline Phospholipides & 0,2 \\
\hline Monoglycérides & 0,1 \\
\hline Diglycérides & 1 \\
\hline Acides gras & 4,6 \\
\hline Triglycérides & 94 \\
\hline
\end{tabular}

Des fragments de tissu mammaire $(63 \mathrm{mg})$, provenant de deux lapines, sont regroupés et incubés pendant une heure dans $2 \mathrm{ml}$ de Krebs Ringer bicarbonaté glucosé. Le milieu d'incubation est récupéré et $5 \mu \mathrm{Ci} / \mathrm{ml}$ de trioléate sont ajoutés pendant une heure à $37^{\circ} \mathrm{C}$ en présence de $95 \%$ de $\mathrm{O}_{2}$ et $5 \%$ de $\mathrm{CO}_{2}$. La radioactivité des différentes classes de lipides dans le milieu est analysée comme précédemment. 


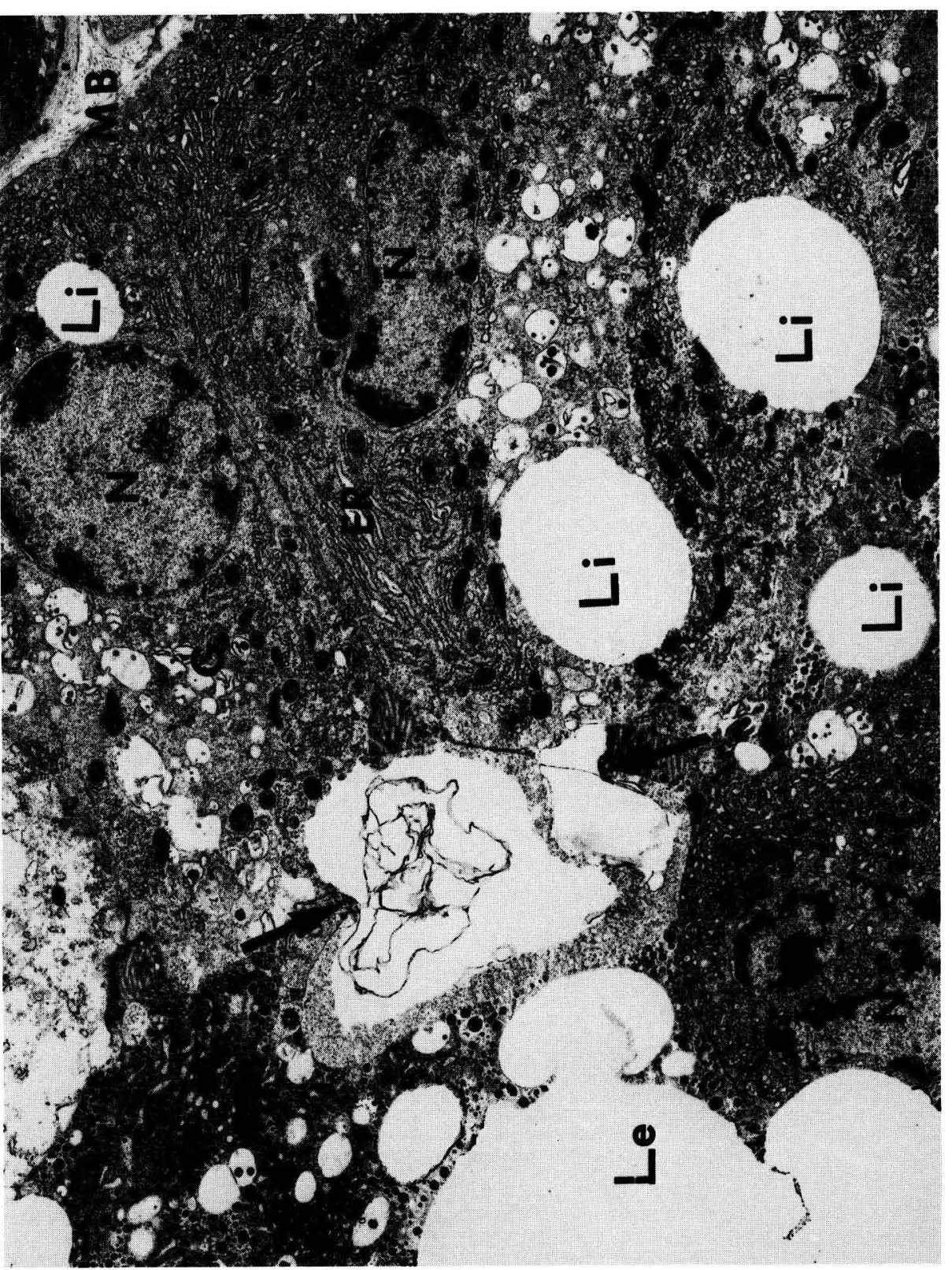

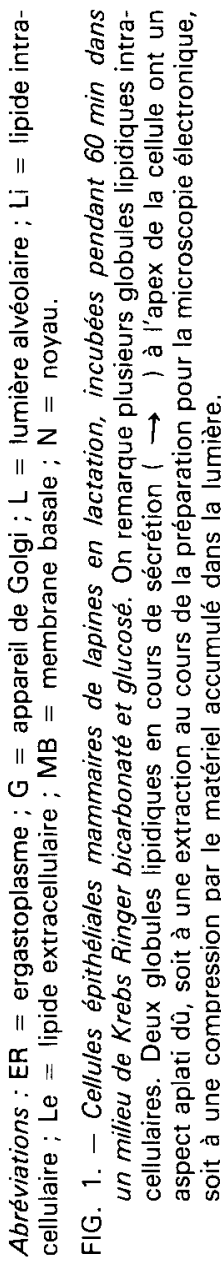




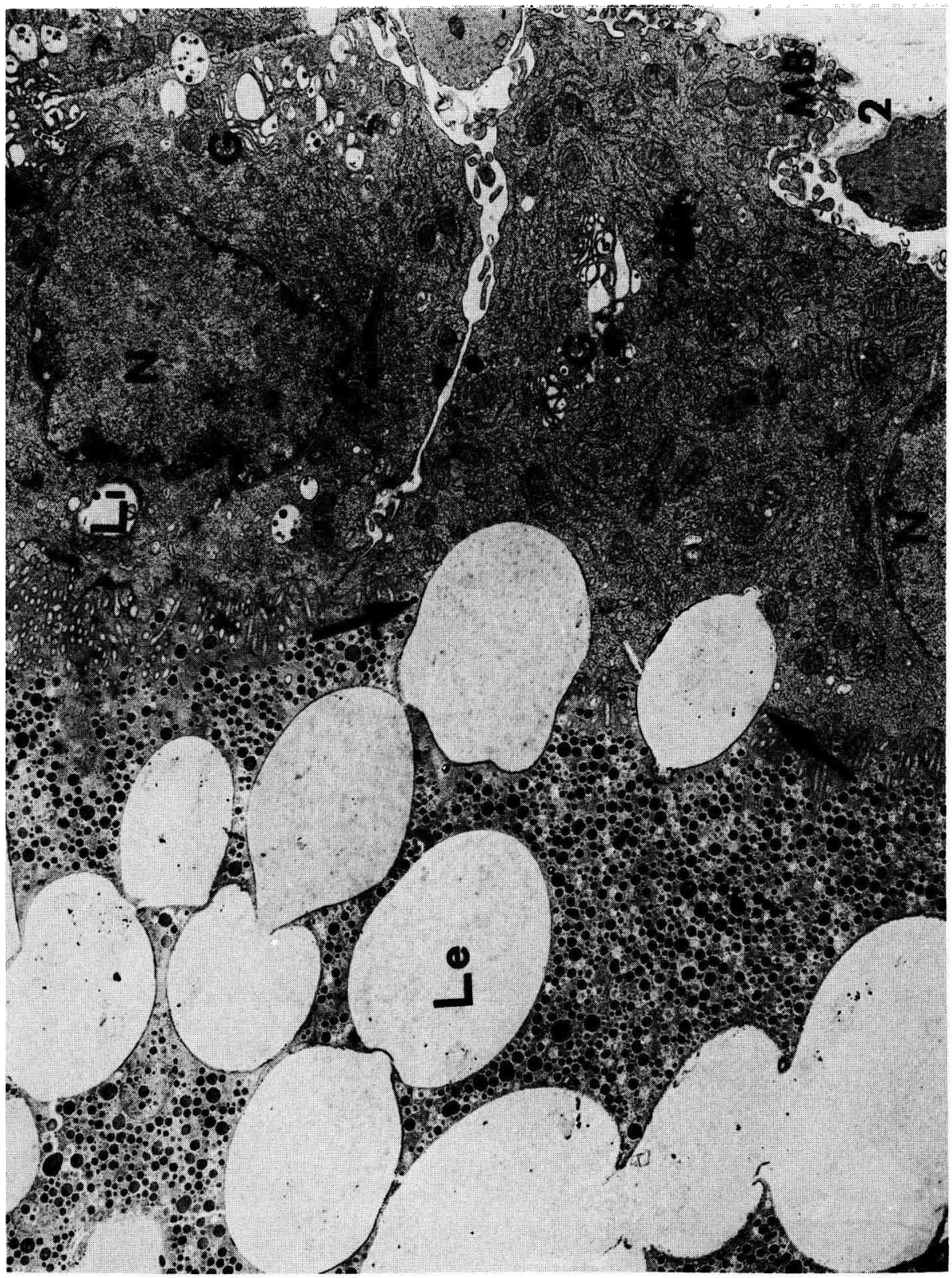

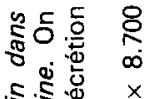

结离

$8 \%$

존

ชั

ฐह 응

ऑ

음흠

용

원

호응

해음

这文

ธ ฮั

w

ฏ 원

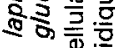

ㅎํ용

象

능

웡 을음

능

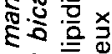

\& す

率

든

웡

义

๖웜

ङે 引

।

ن

은 
Effet de la prolactine sur la répartition des globules lipidiques intra- et extracellulaires. - Le nombre de globules lipidiques situés dans les cellules épithéliales mammaires et dans les lumières des acini en présence ou non de prolactine, a été compté sur les micrographies. Les figures 1 et 2 et le tableau 5 montrent que, après $1 \mathrm{~h}$ d'incubation, les globules lipidiques sont répartis entre l'intérieur des cellules et les lumières des acini. En présence de prolactine, le nombre de globules lipidiques situés dans la lumière est fortement augmenté. II faut de plus remarquer que l'aspect général des cellules témoigne d'une activité sécrétoire accrue en présence de prolactine (cellules denses, état de vidange important).

TABLEAU 5

Répartition des globules lipidiques entre l'intérieur des cellules et la lumière alvéolaire.

\begin{tabular}{lccccc}
\hline & $\begin{array}{c}\text { Nombre } \\
\text { de photos comptés }\end{array}$ & $\begin{array}{c}\text { Globules lipidiques } \\
\text { intracellulaires }\end{array}$ & $\%$ & $\begin{array}{c}\text { Globules lipidiques } \\
\text { contenus } \\
\text { dans la lumière }\end{array}$ & $\%$ \\
\hline Témoin & 120 & 330 & $90,4 \%$ & 35 & $9,6 \%$ \\
\hline Prolactine $(10 \mu \mathrm{g} / \mathrm{ml})$ & 120 & 287 & $73,9 \%$ & 101 & $26 \%$ \\
\hline
\end{tabular}

\section{Discussion.}

Répartition de la radioactivité dans les tissus et dans les milieux. - Les cellules épithéliales mammaires de lapines en lactation incorporent l'acide butyrique ${ }^{14} \mathrm{C}$ dans les lipides. Les différentes classes de lipides sont marquées mais le marquage tissulaire est essentiellement localisé dans les triglycérides. L'activité de la cellule mammaire en lactation est surtout dirigée vers la synthèse des produits de sécrétion. L'incorporation des acides gras dans les triglycérides des lipides tissulaires est bien en accord avec la composition des lipides du lait (97-98\% en poids de triglycérides, Patton et Jensen, 1976).

Nos résultats montrent que la répartition de la radioactivité dans les lipides sécrétés dans le milieu est très différente de celle des lipides intracellulaires : les triglycérides sont relativement moins radioactifs. Les phospholipides, les diglycérides et les acides gras sont plus radioactifs. La sécrétion des lipides s'accompagne donc d'une redistribution parmi les constituants néosynthétisés. Peu de renseignements sont actuellement disponibles sur les propriétés et la composition des globules lipidiques intracellulaires et extracellulaires. Des micro-gouttelettes lipidiques cytoplasmiques, pouvant fournir les triglycérides néosynthétisés aux globules lipidiques, ont été décrites (Dylewski et al., 1984). Des études morphométriques ont montré que les cellules mammaires en lactation contiennent deux populations de gouttelettes de globules lipidiques, des petits globules de moins de $1,5 \mu \mathrm{m}$ de diamètre répartis à l'intérieur des cellules et de plus grands globules 
situés principalement dans la région apicale de la cellule (Stemberger et Patton, 1981). Bien que, en moyenne, les globules lipidiques qui font protrusion dans la lumière soient de grande taille, il n'existe pas d'indication précise sur un ordre de sécrétion en fonction de la taille. Ainsi dans le lait de bovins, $80 \%$ des globules lipidiques sont de petits globules (Walstra, 1969). La grande taille ne paraît donc pas être déterminante pour la sécrétion. On pourrait émettre I'hypothèse que la composition de ces globules plutôt que leur taille donnerait le signal de leur sécrétion et que les modifications de radioactivité des classes de lipides observées seraient liées à ce processus.

Rôle de la LPL sur la modification de répartition de la radioactivité. - La diminution de la radioactivité des triglycérides sécrétés peut être due à une hydrolyse catalysée par la LPL. En présence d'un inhibiteur de cette LPL, le sulfate de protamine, la quantité de triglycérides radioactifs sécrétés est augmentée, ce qui confirme cette hypothèse.

Cette hydrolyse pourrait avoir lieu dans le milieu d'incubation après la sécrétion des globules lipidiques. En effet, parmi les produits de sécrétion de la cellule mammaire, on retrouve des LPL. Ainsi, on sait que des enzymes lipolytiques sont sécrétées dans le lait chez les bovins (Jensen et Pitas, 1976). L'incubation d'un trioléate ${ }^{14} \mathrm{C}$ dans un milieu ayant contenu des fragments de glande mammaire, donc susceptible de contenir des LPL sécrétées, montre que ce triglycéride n'est pas hydrolysé de façon décelable. Ceci suggère que l'hydrolyse par les LPL se produit avant la période d'accumulation dans le milieu. Des LPL ont été mises en évidence dans le tissu mammaire (Clegg, 1981). Leur rôle est essentiellement d'hydrolyser les triglycérides du sérum, libérant les acides gras et les rendant disponibles pour la cellule. Le fonctionnement des LPL est contrôlé par des lipoprotéines provenant du sérum (Clegg, 1980) et il a été proposé qu'une interaction entre ces lipoprotéines et la MFGM pourrait permettre une accessibilité à la LPL et stimuler ainsi son effet. On peut donc émettre l'hypothèse que l'activité des LPL sur l'hydrolyse des triglycérides sécrétés pourrait être favorisée par une proximité entre ces lipoprotéines, les globules lipidiques et les LPL vraisemblablement membranaires, au cours de l'extrusion du globule.

Effet de la prolactine sur la répartition de la radioactivité. - La prolactine provoque une augmentation de la radioactivité relative des triglycérides sécrétés dans les milieux. Cet effet n'est pas modifié par le sulfate de protamine. De plus, les observations morphologiques suggèrent que la prolactine provoque une migration et une libération accélérées dans le milieu. Les effets de la prolactine sur les sécrétions mammaires sont liés à des mouvements de membranes (OllivierBousquet, 1978 ; Kane et al., 1983). Les membranes concernées par ces mouvements sont essentiellement intracellulaires. Toutefois, le trafic intracellulaire augmenté aboutit à une fusion accrue de vésicules golgiennes avec la membrane apicale de la cellule. Cette fusion peut provoquer un accroissement de la surface totale de cette membrane d'une part, et des modifications de sa composition d'autre part. Ces deux modifications pourraient permettre au globule lipidique d'être complètement enveloppé par la membrane plus rapidement, ce qui entraînerait une libération accélérée du globule. Si l'activité des LPL est en relation avec une association entre des protéines qui restent cellulaires et la MFGM, I'envelop- 
pement complet du globule par la membrane, en le séparant de la cellule, pourrait correspondre à une diminution d'activité de l'enzyme. Dans ce cas, en présence de prolactine, les triglycérides des globules lipidiques, du fait de leur transit accéléré, seraient moins sensibles à l'hydrolyse par les LPL.

Un rôle direct de la prolactine sur des systèmes enzymatiques est aussi envisageable. Cependant, les activités de la LPL et des acides gras synthétases sont stimulées par la prolactine durant la lactation (Flint et al., 1981). II serait paradoxal que la prolactine puisse provoquer parallèlement une diminution d'activité des mêmes enzymes, à un pôle différent de la cellule. Seule une compartimentation cellulaire pourrait expliquer ces phénomènes.

En conclusion, nos résultats montrent que la sécrétion des globules lipiques du lait s'accompagne de modifications de la répartition des constituants lipidiques préalablement marqués, qui pourraient être dues à une hydrolyse partielle des triglycérides par la LPL. La prolactine a un effet à la fois sur la quantité et sur la composition des lipides sécrétés.

Reçu en décembre 1985.

Accepté en février 1986.

Remerciements. - Nous remercions Monsieur le Docteur G. Pétrissant pour ses conseils et ses critiques concernant le manuscrit.

\section{Références}

CLEGG R. A., 1980. Activation of milk lipase by serum proteins : possible role in the occurrence of lipolysis in raw bovine milk. J. Dairy Res., 47, 61-70.

CLEGG R. A., 1981. Lipoprotein lipase. Localization on plasma membrane fragments from lactating rat mammary tissue. Biochim. biophys. Acta, 664, 397-408.

CLEGG R. A., 1982. Lipoprotein lipase in the mammary gland and milk. Hannah Res. Inst., Report 1981, ed. Bell and Bain Ltd, Glasgow, pp. 75-87.

COWIE A. T., TINDAL J. S., 1971. In The physiology of lactation. Monogr. Physiol. Soc., 22. Milk Secretion, 136-184.

DAUDET F., AUGERON C., OLLIVIER-BOUSOUET M., 1981. Effet rapide in vitro de la colchicine, du chlorure d'ammonium et de la prolactine sur la sécrétion des lipides du lait dans la glande mammaire. Eur. J. Cell Biol., 24, 197-202.

DYLEWSKI D. P., DAPPER C. H., VALIVULLAH H. M., DEENEY J. T., KEENAN T. W., 1984. Morphological and biochemical characterization of possible intracellular precursors of milk lipid globules. Eur. J. Cell Biol., 35, 99-111.

FLINT D. J., CLEGG R. A., VERNON R. G., 1981. Prolactin and the regulation of adipose tissue metabolism during lactation in rates. Mol. cell. Endocr., 22, 265-275.

FOLCH J., LEES M., SLOANE STANLEY G. M., 1957. A simple method for the isolation and purification of total lipids from animal tissues. J. biol. Chem., 226, 497-509.

JENSEN R. G., PITAS R. E., 1976. Milk lipoprotein lipases : a review. J. Dairy Sci., 59, $1203-1214$.

KANE S., RAYMOND M. N., DUSANTER-FOURT 1., HOUDEBINE L. M., DJIANE J., OLLIVIERBOUSQUET M., 1983. Endocytose de la prolactine dans la cellule épithéliale mammaire : effets des agents lysosomotropes et des inhibiteurs de la transglutaminase. Eur. J. Cell Biol., 30, 244-253. 
KEENAN T. W., DYLEWSKI D. P., 1985. Aspects of intracellular transit of serum and lipid phases of milk. J. Dairy Sci., 68, 1025-1040.

OLLIVIER-BOUSOUET M., 1978. Early effects of prolactin on lactating rabbit mammary gland. Cell Tiss. Res., 187, 25-43.

PATTON S., JENSEN R. G., 1976. Biomedical aspects of lactation. Pergamon Press. STEMBERGER B. H., PATTON S., 1981. Relationships of size, intracellular location and time required for secretion of milk fat droplets. J. Dairy Sci., 64, 422-426.

WALSTRA P., 1969. Studies on milk fat dispersion. II. The globule-size distribution of cow's milk. Netherl. Milk Dairy J., 23, 99. 